\title{
THE LYMPH NODES IN RATS WITH EXPERIMENTAL TYPE 1 DIABETES MELLITUS (DM-1)
}

\author{
Iurii Gavrylenko \\ Department of Paediatric Otorhinolaryngology, Audiology and Phoniatry \\ Shupyk National Medical Academy of Postgraduate Education \\ 9 Dorogozhytska str., Kyiv, Ukraine, 04112 \\ yesyur@ukr.net \\ Margaryta Khomenko \\ Department of Paediatric Otorhinolaryngology, Audiology and Phoniatry \\ Shupyk National Medical Academy of Postgraduate Education \\ 9 Dorogozhytska str., Kyiv, Ukraine, 04112 \\ maasau@gmail.com
}

\begin{abstract}
The aim is to study morphofunctional structure of NALT (nasal associated lymphoid tissue) and visceral lymph nodes of rats with experimental type 1 diabetes mellitus (DM-1) and to define the effectiveness of the treatment with herbal drug "Imupret".

Materials and methods: The experiment involved 20 animals, divided into 4 groups: the $1^{\text {st }}$ group was the control group of healthy rats, the $2^{\text {nd }}$ group was the control group subject to prophylactic treatment with "Imupret, the $3^{\text {rd }}$ group included rats with experimental diabetes, and the $4^{\text {th }}$ group included rats with experimental diabetes subject to treatment with "Imupret. Functional changes in immune organs were evaluated by the results of morphometric analysis; morphological pattern was evaluated by histostructural changes.

Results. The research revealed that under conditions of diabetes mellitus type 1, the volume, area and density of the lymphoid tissue decreased, and only its "fine" cell was detected. The paper demonstrates the development of relative immune deficiency in immunocompetent organs in rats with diabetes mellitus type 1. The use of drug "Imupret" demonstrated its immunomodulatory function, which is especially important in terms of immunosuppression in patient with DM-1.

Conclusion. The received results are of a great clinical significance, and show the necessity of early prevention and treatment of immunity disorders under conditions of diabetes mellitus type 1 .

Keywords: diabetes mellitus type 1, visceral lymph nodes, NALT, immunity histostructural changes, morphometric analysis.

\section{Introduction}

In recent years, within increase of prevalence of immunodeficiency states of different genesis, doctors' interest in the immune system functioning increased significantly [1]. The immune system is known to have a close connection with the endocrine system, and is particularly affected under condition of such disease as diabetes mellitus type 1 (DM-1) [2]. Increased susceptibility to infections in patients with DM-1 is associated with impaired protective functions of the body as a result of suppressed immunity, enhanced cell adhesion of microorganisms, a tendency to catabolic processes. In the pathogenesis of DM-1 and many of its complications, an important place belongs to the violation of the functional capacity of the immune system, which is the subject of numerous studies [3]. In patients with diabetes mellitus, significant changes appears in chemotaxis, a decrease in the bactericidal activity of neutrophils, increased production of reactive oxygen species, leukotrienes, secretion of lysosomal enzymes, and changes in the level of intracellular calcium are often observed [4].

Morphological basis of the immune system is a lymphoid tissue, which is represented by the central and peripheral organs [5]. The structures protecting the mucous membrane are called mucosa associated lymphoid tissue - MALT. MALT consists of visceral lymph nodes of gastrointestinal tract, being the place to induce humoral (antibody mediated) and cellular immunity [6]. The analogue of such tissue in the conducting airway is the nasal-associated lymphoid tissue (NALT) or lymphoid tissue associated with the nasal cavity, which is the regional representative of submucosal of the immune system. It is localized as adjacent to the nasal mucosa and mucosa of the soft palate. NALT is inherent both to humans and rats, but the rats do not have tonsils; thus NALT for rats can be considered as the first immune 
barrier to fight against external antigens in the digestive and respiratory systems [7]. In contrast to the well-characterized Peyer's patches, which are already present in fetal life and seem to be a constitutive part of the integrated mucosal immune system, the tissue genesis of NALT is different [8]. So far no data on NALT formation during embryogenesis in humans are available, but in rats and mice NALT has been observed only postnatal [9]. In fetal human larynges there is only a scattered distribution of immunocompetentcells, but no organized larynx-associated lymphoid tissue (LALT) [10]. Bronchus-associated lymphoid tissue (BALT) is sometimes present in the fetus, but associated mainly with infections, and develops after birth [11]. In the non-infected fetus BALT is found only rarely and in low density [12]. Therefore, the formation of respiratory MALT seems to be a reactive phenomenon in response to microbial stimulation. Dysregulation of mucosal immune responses might be a critical factor in the still unknown etiology of sudden infant death syndrome (SIDS) [13]. No data are available so far on the occurrence of NALT in human adults, as NALT is difficult to investigate in this age group because calcification inhibits adequate sampling and processing of tissue blocks [14]. MALT is characterised by the predominance of local dimeric $\operatorname{IgA}$ production, secreted as secretory $\operatorname{IgA}(\mathrm{s} \operatorname{Ig} \mathrm{A})$ that is responsible for the immune exclusion of bacteria and viruses [15]. The term common mucosal immune system (CMIS) implies that activated lymphocytes derived from one mucosal surface can recirculate and localise selectively in other mucosal surfaces [16]. This connection between different mucosal surfaces permits immunity initiated at one anatomical site to protect other mucosal sites [17]. In rats, NALT is present at birth, earlier than BALT and this is probably due to its strategic position with respect to the incoming air [18]. As rats are obligate nasal breathers, the inspired air, laden with environmental antigens, passes the nasal cavities before it reaches the lungs. [19]. The particular interest from the point of view of clinical effectiveness has the combined herbal preparation BNO 1030 (Imupret) [20].

\section{Aim of research}

To study morphofunctional structure of NALT (nasal associated lymphoid tissue) and visceral lymph nodes of rats with experimental type 1 diabetes mellitus (DM-1) and to define the effectiveness of the treatment with herbal drug "Imupret".

\section{Material and methods}

The research involved intact Wistar male rats (20 animals in total), weighing 130-150 grams, under the conditions of experimental diabetes mellitus type 1. Experimental DM-1 for rats was induced by a single intraperitoneal introduction of Streptozotocin (S0130, Sigma-Aldrich Co. LLC, the USA), dose of $55.0 \mathrm{mg} / \mathrm{kg}$, diluted in $0.1 \mathrm{M}$ citrate buffer, $\mathrm{pH} 4.5$. Rats of the same age in the control group got intraperitoneally $0.5 \mathrm{ml}$ of $0.1 \mathrm{M}$ citrate buffer, $\mathrm{pH} 4.5$. After four weeks of diabetes development, within 14 days, rats got drug Imupret per os $0.05 \mathrm{ml} /$ animal three times a day; chosen concentration meets guidelines for use of the daily dose for children over 12 years and adults, taking into account the factors of species stability for human and rats ( 0.45 and 1.89 respectively). Functional changes in immune organs were evaluated by the results of morphometric analysis. Qualitative histostructural changes were detected (such as appearance of germinal centers in lymphoid tissue nodules, changes in the density of lymphoid tissue); quantitative parameters were subject to statistical evaluation. Micro photographs were obtained using microscope Olympus BX 51. Morphometric analysis was performed with Carl Zeiss software (AxioVision SE64 Rel. 4.9.1), magnification $\times 400$. All rats were divided in 4 groups, 5 animals in each one: the $1^{\text {st }}$ group was the control group of healthy rats, the $2^{\text {nd }}$ group was the control group subject to prophylactic treatment with "Imupret", the $3^{\text {rd }}$ group included rats with experimental diabetes, and the $4^{\text {th }}$ group included rats with experimental diabetes subject to treatment with "Imupret".

\section{Results}

Visceral nodes in the control group of intact rats showed the normal morphological picture. NALT is small and has no signs of immune activation (Fig. 1, $\boldsymbol{a}-\boldsymbol{c})$. In cortical substance (Fig. 1, a) were registered lymphoid follicles, and moderate density lymphocytes were recorded in reticuloendothelial cells. In medulla (Fig. 1, b) was registred a small number of lymphocytes and stromal elements of reticuloendothelial cells. 


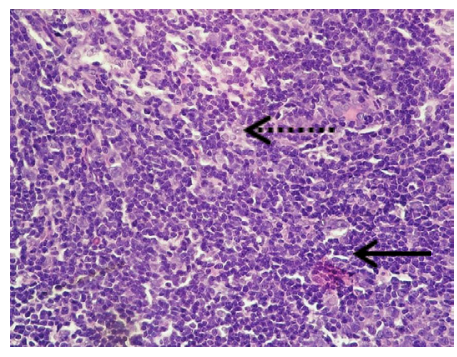

$a$

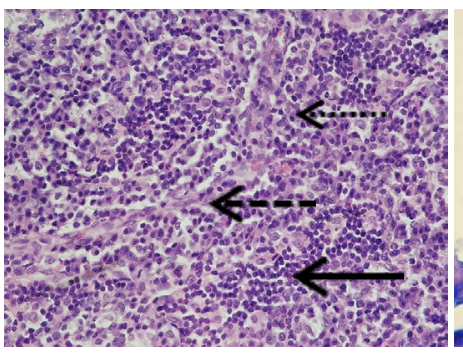

b

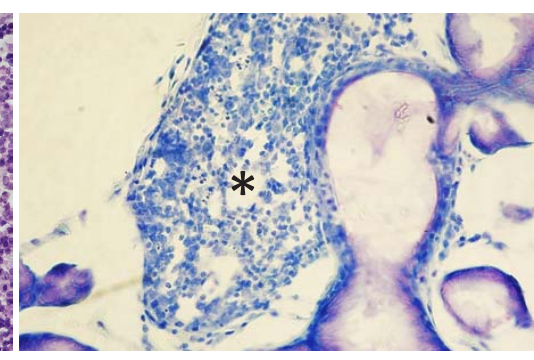

c

Fig. 1. Lymph node of control group: $a$ - cortical substance; $b$ - medulla.

Note: $\longleftarrow-$ lymphocytes; $\ll-\cdots . .-$ - macrophages; $\longleftarrow---$ reticuloendothelial cells. Hematoxylin-eosin; $c$ - Nasal-associated lymphoid tissue (NALT).

* - Clusters of lymphocytes NALT. Toluidine blue

In rats with DM-1 we found the sings of reactive immune activation. In cortex of visceral nodes were registered lymphoid follicles, moderate density lymphocyte, activation of reticuloendotheliocytus, in medulla we found a small number of lymphocytes and stromal elements of reticuloendotheliocytus. In NALT it has been shown significant number of neutrophils and isolated basophils. (Fig. 2, $\boldsymbol{a}-\boldsymbol{c}$ ).

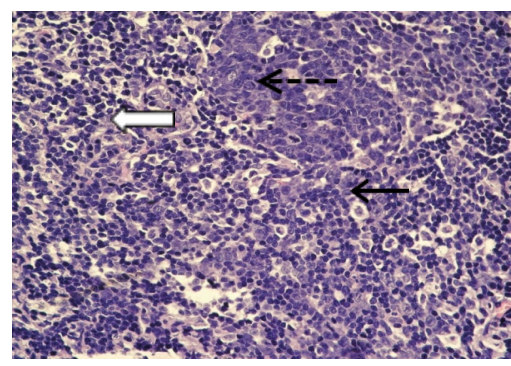

$a$

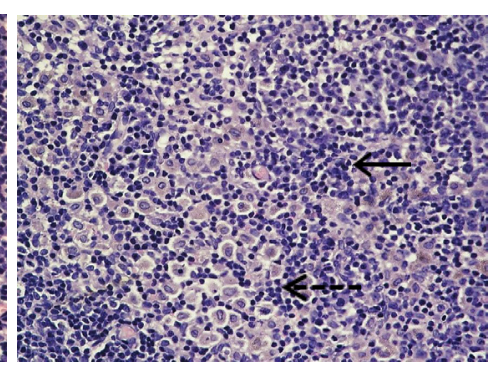

$b$

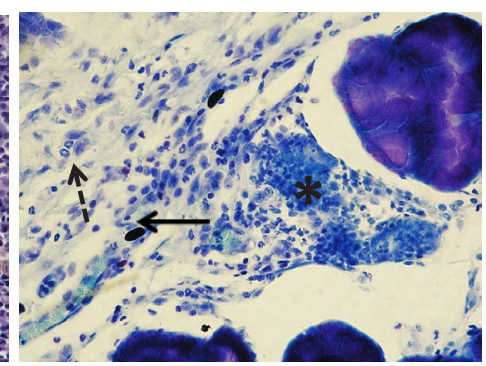

$c$

Fig. 2. Lymph node of diabetic rats. $a$ - cortical substance, $b$ - medulla.

Note: $\longleftarrow-$ lymphocytes; GC - germinal center (lymphoid reaction); $\longleftarrow-$ cell in mitosis; $\leftarrow---$ r eticuloendothelial cells. Hematoxylin-eosin; $c$ - Nasal-associated lymphoid tissue (NALT). Note: $\longleftarrow-$ tissue basophils; $\longleftarrow---$ neutrophils; * - Clusters of lymphocyte NALT. Toluidine blue.

In diabetic rats, which were treated with Imupret, were found the signs of immune response. It was registered the increased density of lymphocytes, activation of mitosis and germinal center (Fig. 3, a, b).

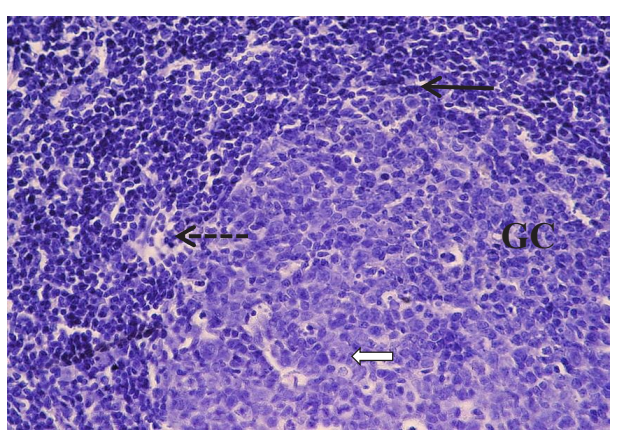

$a$

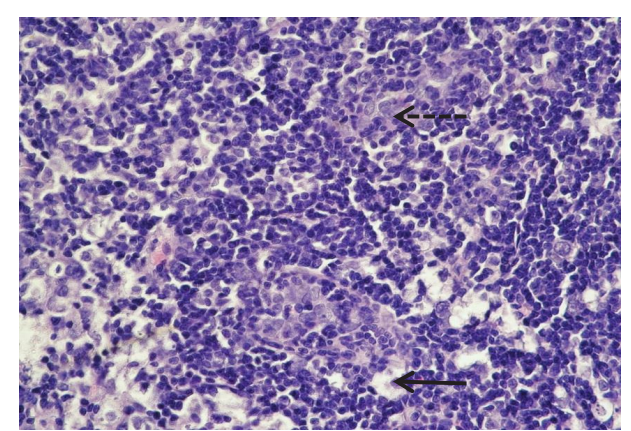

$b$

Fig. 3. Lymph node of diabetic rats, which received Imupret. $a$-cortical substance; $b$ - medulla. Note: $\longleftarrow-$ lymphocytes; GC - germinal center (lymphoid reaction); - cell in mitosis; «--- - reticuloendothelial cells 


\section{Discussion}

Within the study of visceral nodes the control group of intact rats showed no inflammatory changes, signs of antigen presentation. The stromal elements of medulla, brain cords, and capsules showed no signs of reorganization. Only a few macrophages were recorded within the field of view. The volume of NALT was insignificant; in addition, there were recorded the tissue basophils without the signs of degranulation (Fig. 1, $\boldsymbol{a}-\boldsymbol{c}$ ).

Within the study of visceral lymph nodes, the group with diabetes showed reactive changes of lymph node stroma; at morphological level it was manifested in the increasing number of epithelioreticular cells, increase of volume indicators (size of the nuclei and soma). Sinuses of the node showed an increase in the lymphoid cells density, from a moderate increase in the lymphocytes number (especially in individual sinuses) up to occurrence of the secondary nodes with clear germinal centers, being a manifestation of migration of B-lymphocytes and AG-presentation. The cells in mitosis were detected, which could indicate both the migration of immunocompetent cells, and their proliferation. Medullary substance also showed an increase in the lymphoid cells density as well as epithelioreticular cells and occurrence of groups of macrophages. These changes can be assessed as a manifestation of activation of the immune response, and as for the stromal elements, reorganization of the stroma in response to a volume increase of lymphoid tissue representation Fig. 2, $\boldsymbol{a}, \boldsymbol{b}$.

The study of NALT in diabetic group of rats detected only its small focus with accumulation of groups of neutrophils and isolated basophils (Fig. 2, c).

The group of rats with diabetes mellitus, treated with herbal drug "Imupret", showed in the visceral lymph nodes the appearance of significant germinal centers in the cortex of the lymph nodes, significant increase in the lymphocytes density, as well as isolated cells in mitosis. Medullary substance showed the cords of lymphoid cells, increase of lymphocytes density in the dural and portal sinuses (Fig. 3, $\boldsymbol{a}, \boldsymbol{b}$ ).

The results of the research found out that morphological and functional state of lymphoid tissue is closely related to glucose metabolism. It revealed the signs of immunodeficiency among affected rats, both in visceral lymph nodes, and in NALT $(\mathrm{p}<0,05)$. In this group, morphometric analysis showed the increased number of epithelioreticular cells, increase of volume indicators (size of the nuclei and soma). Despite a wide range of antidiabetic drugs used in the clinic of DM-1 and its complications, now more attention is paid to the use of phytotherapy. One of the widely used phytopreparations at present is the Imupret, the mechanism of its action is not completely clarified.

In diabetic group of rats, which received Imupret, we demonstrated increased lymphocytes density in visceral lymph nodes and NALT $(p<0,05)$.

\section{Conclusions}

1. The studied structures, i. e., visceral lymph nodes and NALT refer to the peripheral lymphoid organs; they have a similar structure, shown within the study of their histology.

2. The research demonstrated the changes in these organs in rats with DM-1, induced with Streptozotocin. It revealed the signs of immunodeficiency among affected rats, both in visceral lymph nodes, and in NALT.

3. Use of drug "Imupret" demonstrated its immunomodulatory function $(p<0,05)$, which is especially important in terms of immunosuppression in patient with DM-1.

\section{References}

[1] Daneman, D. (2006). Type 1 diabetes. The Lancet, 367 (9513), 847-858. doi: 10.1016/s01406736(06)68341-4

[2] Muller, L. M. A. J., Gorter, K. J., Hak, E., Goudzwaard, W. L., Schellevis, F. G., Hoepelman, A. I. M., Rutten, G. E. H. M. (2005). Increased Risk of Common Infections in Patients with Type 1 and Type 2 Diabetes Mellitus. Clinical Infectious Diseases, 41 (3), 281-288. doi: 10.1086/431587

[3] Yang, H., Jin, X., Kei Lam, C. W., Yan, S.-K. (2011). Oxidative stress and diabetes mellitus. Clinical Chemistry and Laboratory Medicine, 49 (11), 1773-1782. doi: 10.1515/cclm.2011.250 
[4] Knip, M., Veijola, R., Virtanen, S. M., Hyoty, H., Vaarala, O., Akerblom, H. K. (2005). Environmental Triggers and Determinants of Type 1 Diabetes. Diabetes, 54, S125-S136. doi: 10.2337/diabetes.54. suppl_2.s125

[5] Valle, A., Giamporcaro, G. M., Scavini, M., Stabilini, A., Grogan, P., Bianconi, E. et. al. (2013). Reduction of Circulating Neutrophils Precedes and Accompanies Type 1 Diabetes. Diabetes, 62 (6), 2072-2077. doi: 10.2337/db12-1345

[6] James, S., Perry, L., Gallagher, R., Lowe, J., Dunbabin, J., McElduff, P. et. al. (2014). Service usage and vascular complications in young adults with type 1 diabetes. BMC Endocrine Disorders, 14 (1). doi: 10.1186/1472-6823-14-39

[7] Szablewski, L., Sulima, A. (2016). The structural and functional changes of blood cells and molecular components in diabetes mellitus. Biological Chemistry, 398 (4), 411-423. doi: 10.1515/hsz-2016-0196

[8] Guzman-Bautista, E. R., Ramirez-Estudillo, M. C., Rojas-Gomez, O. I., Vega-Lopez, M. A. (2015). Tracheal and bronchial polymeric immunoglobulin secretory immune system (PISIS) development in a porcine model. Developmental \& Comparative Immunology, 53 (2), 271-282. doi: 10.1016/j.dci.2015.07.010

[9] Thibeault, S. L., Rees, L., Pazmany, L., Birchall, M. A. (2009). At the crossroads: mucosal immunology of the larynx. Mucosal Immunology, 2 (2), 122-128. doi: 10.1038/mi.2008.82

[10] Bertoni, A. G., Saydah, S., Brancati, F. L. (2001). Diabetes and the Risk of Infection-Related Mortality in the U.S. Diabetes Care, 24 (6), 1044-1049. doi: 10.2337/diacare.24.6.1044

[11] Casteleyn, C., Broos, A. M. C., Simoens, P., Van den Broeck, W. (2010). NALT (nasal cavity-associated lymphoid tissue) in the rabbit. Veterinary Immunology and Immunopathology, 133 (2-4), 212-218. doi: 10.1016/j.vetimm.2009.08.011

[12] Guzik, T. J. (2002). Mechanisms of Increased Vascular Superoxide Production in Human Diabetes Mellitus: Role of NAD(P)H Oxidase and Endothelial Nitric Oxide Synthase. Circulation, 105 (14), 1656-1662. doi: 10.1161/01.cir.0000012748.58444.08

[13] Roux, M. E., del Lopez, M. C., Florin-Christensen, A. (2000). Mucosal Immunity. Probiotics 3, 12-28. doi: 10.1007/978-94-017-2768-6_1

[14] Heritage, Brook, Underdown, McDermott (2001). Intranasal immunization with polymer-grafted microparticles activates the nasal-associated lymphoid tissue and draining lymph nodes. Immunology, 93 (2), 249-256. doi: 10.1046/j.1365-2567.1998.00420.x

[15] Kraal, G. (2005). Nasal-Associated Lymphoid Tissue. Mucosal Immunology, 415-422. doi: 10.1016/b978-012491543-5/50027-9

[16] Sosa, G. A., Roux, M. E. (2004). Development of T Lymphocytes in the Nasal-associated Lymphoid Tissue (NALT) from Growing Wistar Rats. Clinical and Developmental Immunology, 11 (1), $29-34$. doi: 10.1080/10446670410001670463

[17] NALT / BALT and Oral Cavity (2009). Mucosal Immunology, 2 (1s). doi: 10.1038/mi.2009.43

[18] Sepahi, A., Salinas, I. (2016). The evolution of nasal immune systems in vertebrates. Molecular Immunology, 69, 131-138. doi: 10.1016/j.molimm.2015.09.008

[19] Hathaway, L. J., Kraehenbuhl, J.-P. (2000). The role of M cells in mucosal immunity. Cellular and Molecular Life Sciences, 57 (2), 323-332. doi: 10.1007/p100000693

[20] Berger, T. (2008). Tolerability and efficacy of a herbal combination preparation in children and adolescents with recurrent infections of the upper respiratory tract. MMW Fortschr Med., 150, 85-90. 